\title{
Impact of nitrogen on effective work function of Metal/Oxide stack: a first-principles study of the $\mathrm{Pt} / \mathrm{HfO}_{2}$ interface
}

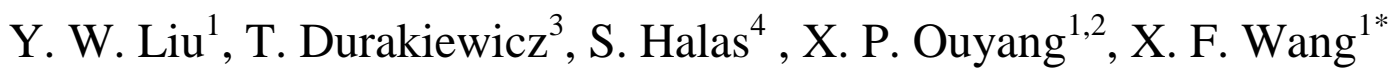 \\ ${ }^{1}$ School of Materials Science and Engineering, Xiangtan University, Hunan411105, \\ China \\ ${ }^{2}$ Northwest Institute of Nuclear Technology, P.O. Box 69-9, Xi'an, Shanxi 710024, China \\ ${ }^{3}$ Los Alamos National Laboratory, Mailstop K764, Los Alamos, New Mexico 87545, USA \\ ${ }^{4}$ Institute of Physics, Maria Curie-Sklodowska University, Lublin 20-031, Poland
}

*Author to whom correspondence should be addressed. Email: onexf@xtu.edu.cn;

Phone: 86-732-58298518; Fax: 86-732-58298518 


\begin{abstract}
Modulating the effective work function $\left(\Phi_{\text {eff }}\right)$ of metal electrodes is both crucial and challenging in metal-oxide-semiconductor transistors. The introduction of atomic dopants is shown to be an effective method to modify the interface $\Phi_{\text {eff. }}$. Here we apply the first principles calculations to systematically explore the impact of incorporation of the non-metallic ambient gas element $\mathrm{N}$ on the effective work function of a $\mathrm{Pt} / \mathrm{HfO}_{2}$ interface. We find that $\Phi_{\text {eff }}$ is very closely related to the doped atomic position and concentration of the incorporated $\mathrm{N}$ atoms. The incorporation is favored at the interface near $\mathrm{HfO}_{2}$ side, and it can significantly increase the interface $\Phi_{\text {eff }}$ for the $\mathrm{Pt} / \mathrm{HfO}_{2}$ stack (making it more $p$-type). Also, the $\Phi_{\text {eff }}$ increases with the increase of doped $\mathrm{N}$ concentration due to the mitigation effect of doped $\mathrm{N}$ on interface dipole moment. Our findings will provide guidance in developing the environment annealing approach to modulation of $\Phi_{\text {eff }}$ of the Metal/Oxide stack.
\end{abstract}

Key words: A. Metal/Oxide interface; B. N dopant; D. Work function; E. First-principles study;

\title{
1. Introduction
}

Metal/metal oxide interfaces play an important role in several fields of modern science and technology, such as catalysis or coatings, and are central in the complementary metal-oxide-semiconductor (CMOS) process. Propelled by Moore's law, the transistor scaling race continues relentlessly for decades, and innovations in gate stack have been a crucial part of the success of the semiconductor technology [1-2]. In order to continue this development, and orchestrated effort is needed towards battling the leakage current problem, where the replacement of $\mathrm{SiO}_{2}$ with high dielectric constant gate dielectric and the poly-silicon electrode with a metal gate promises to provide a suitable a solution [3].

As a transition metal with unique properties, platinum has high work function, high chemical stability, and strong catalytic activity. Hf-based oxides (e.g., $\mathrm{HfO}_{2}$ ) exhibit the best combination of desired properties and have been successfully introduced into the 45-nm technology node along. Thus, the $\mathrm{Pt} / \mathrm{HfO}_{2}$ stack is generally used as supported 
catalysts and as a promising metal gate/high- $k$ dielectric combination in advanced CMOS. The work function of a metal electrode needs to be optimized in such a way that the metal Fermi level lines up with either the valence band maximum or conduction band minimum of the underlying Si substrate. However, it was experimentally shown that the platinum work function is high, varying between 4.6 and $4.9 \mathrm{eV}$ in the p-type CMOS device, different from its vacuum work function $(\sim 5.6 \mathrm{eV})$, due to several interfacial effects (such as charge transfer, bond formation, defect accumulation, dipole creation, etc.) [4]. Generally speaking, the vacuum level discontinuity across the interface implies that the work function of the metal on the surface may be different from that of the interface. The latter is generally known as the effective work function $\left(\Phi_{\text {eff }}\right)$, which depends on the surface work function of metal $(\Phi)$ as well as the additional dipoles generated at the metal and oxide interface [5]. Therefore, the $\Phi_{\text {eff }}$ is a critical parameter in designing the metal electrodes, and it strongly depends on the processing conditions (e.g., the oxygen and nitrogen partial pressure during annealing) to which the device is subjected.

It has been widely reported that $\Phi_{\text {eff }}$ of metals is strongly related to the processing conditions, and the $\Phi_{\text {eff }}$ of many metals is shifted toward the middle of the Si band gap upon high temperature annealing, regardless of the initial value of the vacuum work function [6]. Experiment and theory suggest possible ways of tuning $\Phi_{\text {eff }}$ by implanting dopants into the gate stack, or by introducing an interfacial "capping" layer either at the $\mathrm{Si} / \mathrm{HfO}_{2}$ interface or at the metal/ $\mathrm{HfO}_{2}$ interface [7]. There are many reported ways of tuning the $\Phi_{\text {eff }}$ of gate electrodes, for example, by dopants implantation and annealing, which induces the variation of the metal-dielectric interface status, which leads to the modulation of the work function of the gate electrode [8]. For example, such tuning can be achieved by inserting a capping layer with a dipole moment pointing from dielectric to metal at metal/ $/ \mathrm{HfO}_{2}$ interfaces or pointing from $\mathrm{Si}$ to dielectric at $\mathrm{Si} / \mathrm{HfO}_{2}$ interfaces [9], or by implanting $\mathrm{Al}$ into TiN electrode by diffusion [10]. The $\mathrm{Pt} / \mathrm{HfO}_{2}$ interface stack is usually annealed in forming gas or ambient atmosphere which contains $\mathrm{N}_{2}$, especially in the post metallization anneal (PMA) process of gate-first CMOS integration scheme [11]. The role of oxygen at the $\mathrm{Pt} / \mathrm{HfO}_{2}$ interface has been researched extensively [12], but 
how nitrogen impacts the metal/metal oxide interface remains unknown.

Here we describe the results of our theoretical exploration of the dependence of the metal/High- $k$ interface properties on the atmosphere treatment conditions. We propose that effective work function adjustment can be obtained this way. In this work, we have adopted the density functional theory calculations to explore the impact of element $\mathrm{N}$ on the $\Phi_{\text {eff }}$ of $\mathrm{Pt} / \mathrm{HfO}_{2}$ stack in an ambient annealing atmosphere, and we have determined the variation in morphology of the $\mathrm{Pt} / \mathrm{HfO}_{2}$ interface with implantation of elemental nitrogen.

\section{Method}

Our work function calculations were performed using the Vienna ab initio Simulation Package (VASP) based on the density functional theory (DFT) [13] and the projector-augmented wave (PAW) pseudo-potential [14]. The exchange-correlation function was treated within the Perdew, Burke and Ernzerhof (PBE) [15]. The total energy was converged to within $1 \mathrm{meV}$ with a kinetic energy cutoff of $400 \mathrm{eV}$. Monkhorst-Pack k-point meshes are adopted, i.e., $11 \times 11 \times 11$ and $15 \times 15 \times 15$ for interface structure relaxation and static calculation, respectively. All calculations assume no spin polarization and are performed using the 'accurate' setting within VASP to avoid wraparound errors. We choose monoclic phase (m-) $\mathrm{HfO}_{2}$ as a model structure, and the calculated lattice constants for bulk $m$ - $\mathrm{HfO}_{2}$ are $a=5.136 \AA, b=5.195 \AA, c=5.307 \AA$ and $\beta=99.76^{\circ}$, which is about $2 \%$ higher than the experimental value of $a=5.116 \AA, b=$ $5.172 \AA, c=5.295 \AA$ and $\beta=99.18^{\circ}$, due to the GGA error [16]. The calculated lattice

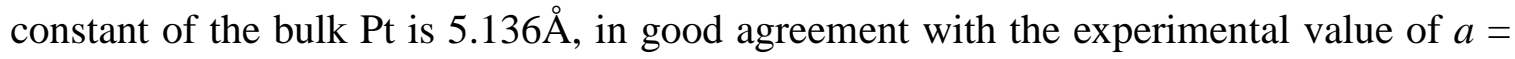
$5.128 \AA$ [17], Next, we choose $(1 \times 1)$ unit cell of $\mathrm{HfO}_{2}(001)$ surface interfaced with $(2 \times \sqrt{ } 3)$ unit cell of Pt surface (111) surface orientations. To match the lateral periodicity, the requirement of least strain for interface needs to be taken into account. i.e., Pt surface is stretched to match $\mathrm{HfO}_{2}$ surface, and $-5.2 \%$ and $8.3 \%$ strain emerges alone $a$ and $b$ directions of $\mathrm{HfO}_{2}$, respectively. Such a strain state is considered appropriately, because Pt is to be deposited on a $\mathrm{HfO}_{2}$ film in the actual production process and the Pt layer is expected to conform to the $\mathrm{HfO}_{2}$ layer. Our interface model was constructed in a 
super-cell that consist of $\mathrm{Pt} / \mathrm{HfO}_{2}$ hetero-structure and a vacuum of $10 \AA$. All atoms were allowed to relax to their equilibrium positions. To determine the preferred configuration of $\mathrm{N}$ dopant at the near-interface area, as well as its segregation into the oxide or the metal slab, we calculated the corresponding interface formation energy. The lower interface formation energy means the structure is more thermodynamically stable. The formation energy ( $\left.E_{\text {form}}\right)$ is general a function of chemical potential and can be estimated by [18], $E_{\text {form }}=\left[E_{\text {doped }}-E_{\text {pure }}-n\left(E_{\mathrm{N}}-E_{\mathrm{M}}\right)\right] / A$, where $E_{\text {doped }}$ is the total energy of the doped super-cell configuration, $n$ is the number of atoms of element $N$ in the super-cell, $E_{\text {pure }}$ is the total energy of undoped super-cell, and $\mathrm{E} x$ and $\mathrm{E} M$ are respective the chemical potential of doping element and the substituted element [19]. Subsequently, the methodology to determine $\Phi_{\text {eff }}$ is supposed to discuss. The effective work function can be derived by using the interface dipole moment for the structure identified above and the $\mathrm{Pt}$ vacuum work function $(\Phi)$, which can be expressed as [6],

$$
\Phi_{\mathrm{eff}}=\Phi+\Delta \Phi=\Phi+4 \pi \Delta \mu / A
$$

where $A$ is the interface area, and $\Delta \mu$ is the extra dipole moment generated at the interface when metal contacts dielectric (oxide). Because Pt surface is stretched to match $\mathrm{HfO}_{2}$ surface, we should take vacuum work function of strained Pt surface as $\Phi$. The calculated $\Phi$ for the strained (111) Pt surface (required to form a coherent interface with $\mathrm{HfO}_{2}$ as described above) is $5.72 \mathrm{eV}$ in this work and corresponding value for the Pt slab equilibrium is $5.68 \mathrm{eV}$ (consistent with experimental value of $5.65 \mathrm{eV}$ ), which is according with the $\Phi$-strain relationship was reported in our previous work [20].

\section{Results and Discussions}

\section{A. Configurations concerned}

To understand energetics of $\mathrm{N}$ dopant within the $\mathrm{Pt} / \mathrm{HfO}_{2}$ hetero-structure, we've performed separate calculations for different interface configurations. The relaxed $\mathrm{Pt} / \mathrm{HfO}_{2}$ structure is shown in Figure 1. It's noted that the $\mathrm{HfO}_{2}$ side for the clean interface in present work is fully $\mathrm{O}$ terminated, which is mainly formed by $\mathrm{Pt}-\mathrm{O}$ bonds with bond lengths ranging from 1.735 angstrom to 2.009 angstrom. 


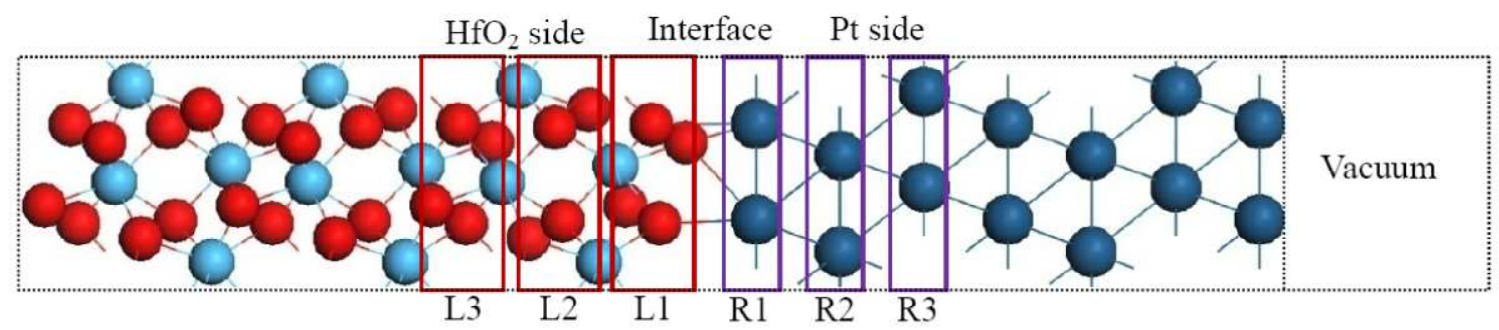

FIG.1. The model Interface of $\mathrm{Pt} / \mathrm{HfO}_{2}$ heterostructure. $\mathrm{HfO}_{2}$ side layers of $\mathrm{O}$ atoms (left) and $\mathrm{Pt}$ side layers of Pt atoms (right) substituted by N atoms are noted as L1-L3 and R1-R3, respectively.

In order to describe morphology of the $\mathrm{Pt} / \mathrm{HfO}_{2}$ with element $\mathrm{N}$ dopant implantation, we consider two basic configurations (see Fig. 1), e.g., substituting $\mathrm{N}$ for $\mathrm{O}$ atoms on the dielectric side (No) and substituting $\mathrm{N}$ for $\mathrm{Pt}$ atoms on the metal side ( $\mathrm{NPt}$ ), and model the various interface morphologies by varying the concentrations and doped positions. In each case the atomic positions were relaxed and the in-plane lattice constants and all angles are kept fixed. Next, we further construct the difference models for each configuration (as illustrated in Fig. 1), i.e., (1) a clean $\mathrm{Pt} / \mathrm{HfO}_{2}$ interface (referred as a clean model), and when the doped layer is (2) the first interface layer (L1 or R1 model), (3) the second layer (L2 or R2 model), and (4) the third layer (L3 or R3 model) of the metal or dielectric side, respectively. In the case of No-L1 model, the $\mathrm{N}$ atoms are supposed to be fourfold coordinated and segregate the interface layer, which are bonded to $2 \mathrm{Pt}$ and $2 \mathrm{Hf}$ atoms. Due to the larger atomic radius of $\mathrm{N}$ relative to $\mathrm{O}$, the $\mathrm{N}$ atoms move slightly closer to the interface deviating from original $\mathrm{O}$ position, and the lengths of $\mathrm{Pt}-\mathrm{N}$ bonds ranging from 1.925 angstrom to 2.313 angstrom.

\section{B. Formation energy of $N$ at the interface}



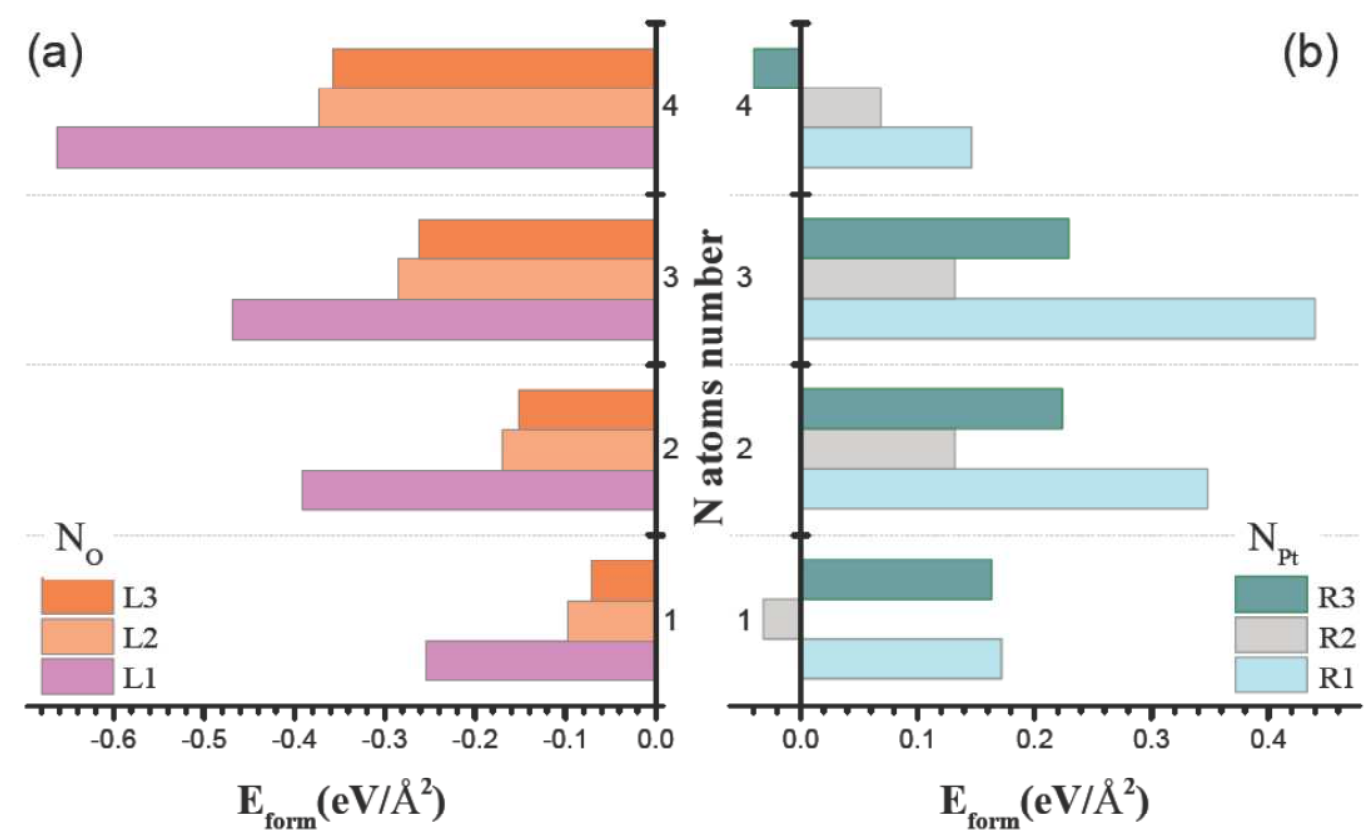

FIG. 2. Formation energy per interface area (in $\mathrm{eV} / \AA 2$ ) of $\mathrm{N}$ doped $\mathrm{Pt} / \mathrm{HfO}_{2}$ heterostructure as a function of dopant atoms number with respect to undoped system. (a) No configurations concerned with L1-L3 Models. (b) NPt configurations concerned with R1-R3 Models.

The formation energies for doped hetero-structures (with respect to that of a perfect system) as a function of $\mathrm{N}$ dopant atoms number are plotted in Fig. 2. In the case of No configuration (Fig.2a), formation energies are negative and decrease dramatically with the increase of $\mathrm{N}$ concentrations. The formation energy of L1 model decreases more significantly in comparison with the other models, which means nitrogen atoms are energetically inclined to segregate into the $\mathrm{Pt} / \mathrm{HfO}_{2}$ interface. However, in the case of $\mathrm{NPt}$ configuration (Fig.2b), formation energy increases firstly then decreases with the increase of $\mathrm{N}$ concentrations, and most values of $E_{\text {form }}$ are positive. It indicates that $\mathrm{N}$ is very difficult to diffuse to $\mathrm{Pt}$ side of stack. Therefore, nitrogen atoms prefer to segregate the interface by substituting $\mathrm{O}$ atoms in $\mathrm{HfO}_{2}$ side, which is mostly due to fact that the radius and electro-negativity of nitrogen are much closer to those of oxygen compared with platinum. Then, we will employ the No configuration to investigate the effective work function of $\mathrm{N}$ doped $\mathrm{Pt} / \mathrm{HfO}_{2}$ stack in the following sections.

\section{Impact of interface $N$ on the effective work function}

As discussed above, due to interface effect, the actual work function doesn't always correspond to vacuum work function in experimental observation [21]. This work 
function shift $(\Delta \Phi)$ can be defined by the difference of the effective work function of stack and the vacuum work function of corresponding metal. We can rewrite equation (1) as, $\Delta \Phi=\Phi_{\text {eff }}\left(\mathrm{Pt} / \mathrm{HfO}_{2}\right)-\Phi(\mathrm{Pt})=4 \pi \Delta \mu / A$, where $\Phi\left(\mathrm{Pt} / \mathrm{HfO}_{2}\right)$ and $\Phi(\mathrm{Pt})$ are the work function of the interface system and Pt surface slab, respectively.

Considering unstable NPt configuration, we adopt No configuration to investigate the work function for $\mathrm{HfO}_{2} / \mathrm{Pt}$ system. $\mathrm{N}$ dopant induced changes in the work function shift $(\Delta \Phi)$ as a function of $\mathrm{N}$ atoms number (i.e., $\mathrm{N}$ dopant concentration) is shown in Figure 3. We found that $\Delta \Phi$ is strongly correlated with $\mathrm{N}$ concentrations and doped positions (doped layers). Firstly, the work function shift for No configuration for all models (including clean and L1-L3 models) are negative, which indicates $\Phi_{\text {eff }}$ of stacks is less than $\Phi$ of Pt surface, and the absolute value of work function shift $(|\Delta \Phi|)$ decreases with the increase of $\mathrm{N}$ concentration (i.e., the $\Phi_{\text {eff }}$ decreases with the increase of $\mathrm{N}$ concentration). Specifically, the $\Delta \Phi$ undergoes a drastic increase in comparison to undoped system when one $\mathrm{N}$ atom is doped. Next the increasing trend becomes much more smooth and steady when doped $\mathrm{N}$ is increased to two, three and four atoms, respectively. Secondly, the presence of $\mathrm{N}$ atoms in the $\mathrm{L} 1$ model has a significant effect on the work function shift. As seen in the inset in Fig. 3, replacing one $\mathrm{O}$ atom by $\mathrm{N}$ atom in the $\mathrm{L} 1$ model results in a decrease of about $0.2 \mathrm{eV}$ or $75 \%$ in the $|\Delta \Phi|$ compared to the clean model. When doped $\mathrm{N}$ atoms are gradually far away from the interface (the $\mathrm{N}$ is placed in the second L2 or third L3 layer), their value of work function does not show any obvious shifts. Above results will provide experimentalists with some insights in engineering the $\Phi_{\text {eff }}$ (or $\Delta \Phi$ ) by controlling the proportion of $\mathrm{N}_{2}$ and $\mathrm{O}_{2}$ of the annealing atmosphere. This results are consistent with the prior experimental observations in which $\mathrm{Pt} / \mathrm{HfO}_{2}$ stack after annealing in air or $\mathrm{N}_{2}$ atmosphere displays a larger $\Phi_{\text {eff }}$ than that as-deposited state [22], and indicates $\mathrm{N}$ is an efficient dopant for $p$-type MOSFETs. 


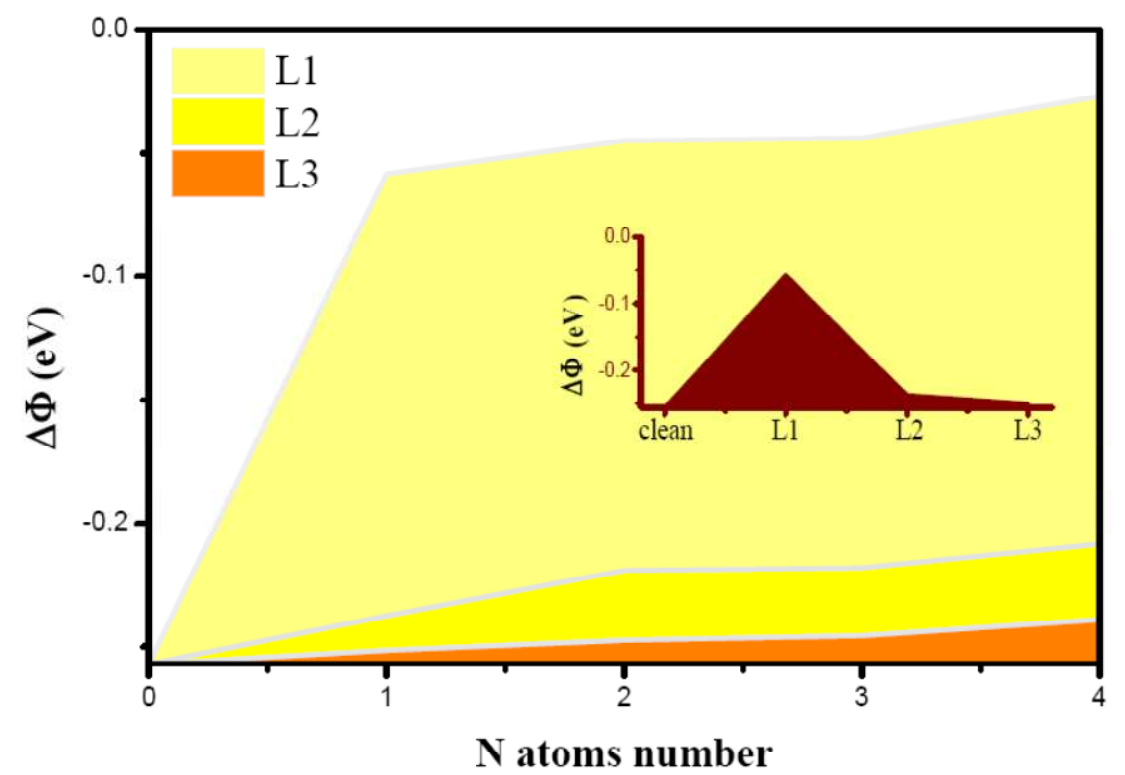

FIG. 3. $\mathrm{N}$ dopant induced changes in work function shift $(\Delta \Phi$, in $\mathrm{eV})$ as a function of $\mathrm{N}$ concentration. Inset: the $\Delta \Phi$ of the four types of interface models: clean, L1, L2 and L3.

To examine the origin of this shift, we try to establish the relationship between the work function shift and interface dipole moment as well as electron density distribution. According to equation 1 , the $\Delta \Phi$ is proportional to interface normal component of extra dipole moment $(\Delta \mu)$ induced by interface effect, and the $\Delta \mu$ of interface system $\mu\left(\mathrm{Pt} / \mathrm{HfO}_{2}\right)$ can be given as [21], $\Delta \mu=\mu\left(\mathrm{Pt} / \mathrm{HfO}_{2}\right)-\mu\left(\mathrm{HfO}_{2}\right)-\mu(\mathrm{Pt})$, where $\mu\left(\mathrm{Pt} / \mathrm{HfO}_{2}\right), \mu\left(\mathrm{HfO}_{2}\right)$ and $\mu(\mathrm{Pt})$ are surface normal components of dipole moments of interface, $\mathrm{HfO}_{2}$ substate, and $\mathrm{Pt}$ layer respectively. The $\Delta \mu$ of dopted system is determined by the electronic interaction between $\mathrm{N}$ and other atoms in $\mathrm{Pt} / \mathrm{HfO}_{2}$ interface, which can be derived as [23], $\Delta \mu=-\int_{\mathrm{z}_{0}}^{\mathrm{z}_{0}+l / 2} z \Delta \rho(z) \mathrm{d} z$, where $z$ direction is perpendicular to the interface plane, $z_{0}$ is chosen to be at the center of $\mathrm{HfO}_{2}$-substrate side, and $l$ is the length of the stack in the $z$ direction. The $\Delta \rho$ is the charge density difference averaged over the plane parallel the surface at some position $z$, described by, $\Delta \rho(z)=$ $\rho(z)_{\mathrm{Pt} / \mathrm{HfO}_{2}}-\rho(z)_{\mathrm{Pt}}-\rho(z)_{\mathrm{HfO}_{2}}$, where $\rho(z)_{\mathrm{Pt} / \mathrm{HfO}_{2}}$ is the charge density of the interface system. $\rho(z)_{\mathrm{Pt}}$ and $\rho(z)_{\mathrm{HfO}_{2}}$ are the charge densities of the isolated clean $\mathrm{Pt}$ layer and $\mathrm{HfO}_{2}$ substrate respectively, both of which are in the precise positions adopted at their corresponding interface system [24]. 


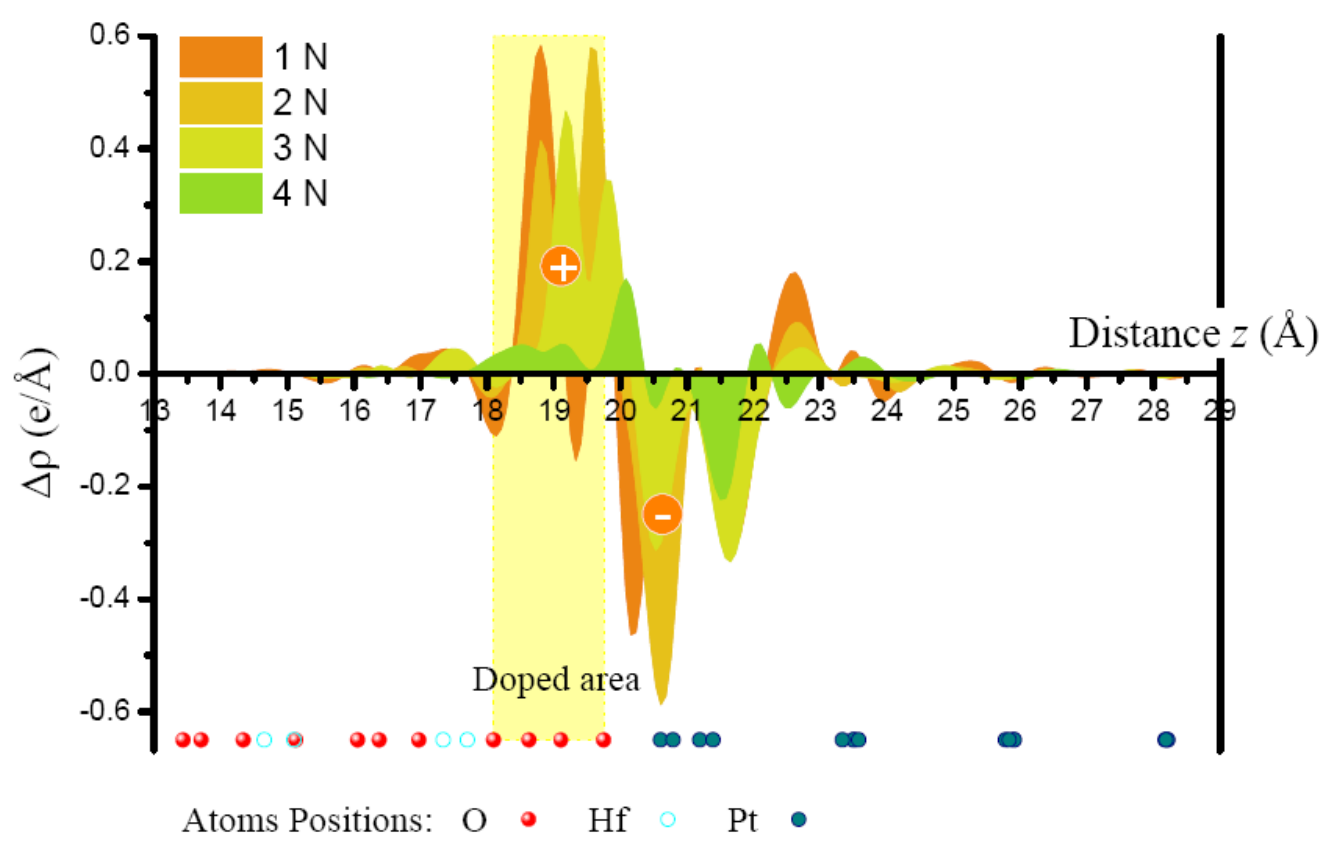

FIG. 4. Charge density difference of No-L1 configuration with different concentrations as a function of the direction perpendicular $z$ to the interface. The positions of atoms are listed by circles in the bottom, and doped area in $\mathrm{HfO}_{2}$ side is drawn by yellow.

By analyzing the charge density difference (Eq. 7), we can explain the mechanism of $\Delta \Phi_{\text {eff }}$ shifts in terms of extra dipole moment at the interface. For a given metal/dielectric stack, extra interface dipoles are formed in the interface region due to electron transferring from Pt side to dielectric $\left(\mathrm{HfO}_{2}\right)$ side. Figure 4 displays the distributions of $\Delta \rho$ for No-L1 model with different concentrations as a function of the direction perpendicular $z$ to the interface. Clearly, the interaction of $\mathrm{N}$ with $\mathrm{Pt} / \mathrm{HfO}_{2}$ is noticeable. Firstly, the interface dipole is mainly due to the contribution of the first atom layer (L1), while the second layer (L2) has a very small contribution to the interface dipole and the effects of the third (L3) as well as farther layers are negligible. This result explains that L1 model exhibits most significant effect on the work function shift in the L1-L3 models. Furthermore, it is evident from Fig. 4 that the amplitude of $\Delta \rho$ decreases when doped-N concentration increases, i.e., diffusion of $\mathrm{N}$ element into the interface mitigates the electrons transfer to some extent. It may be attributed to the element electro-negativity difference. We found the electronegativity of N, O, Hf and Pt by Pauling scale are 3.04, 3.44, 1.3 and 2.28, respectively, and electronegativity of $\mathrm{N}$ locates between $\mathrm{O}$ and $\mathrm{Pt}$ [17]. The electron charges transfer from $\mathrm{Pt}$ to $\mathrm{O}$ when $\mathrm{Pt}$ layer contacts $\mathrm{HfO}_{2}$ layer and forms an interface, while the electrons transferring will be weakened when $\mathrm{O}$ atoms are 
gradually replaced by $\mathrm{N}$ atoms, which is much less electronegative than $\mathrm{O}$. As a result, the $\mathrm{N}$ incorporation can deplete the electron charge transfer in $\mathrm{Pt} / \mathrm{HfO}_{2}$ interface, and interface dipole moment decreases correspondingly, there by the value effective work function (or work function shift) would be raised.

\section{Summary}

In summary, we have investigated the impact of gas ambient element $\mathrm{N}$ on the $\mathrm{Pt} / \mathrm{HfO}_{2}$ interface effective work function $\left(\Phi_{\text {eff }}\right)$ as well as work function shift $(\Delta \Phi)$ using density functional theory calculations. First, we have found that doped ambient gas element $\mathrm{N}$ tends to be favored at the interface and near the dielectric side, the system stability decreases with increasing dopant concentration. Second, work function shift of $\mathrm{Pt} / \mathrm{HfO}_{2}$ stack is strongly correlated to doped $\mathrm{N}$ position and concentration, i.e., the modulation of $\Phi_{\text {eff }}$ and $\Delta \Phi$ is most effective when the first atom layer in $\mathrm{HfO}_{2}$ side is doped, and the work function shift decreases (more $p$-type) with an increase of doped concentration. Third, the $\mathrm{N}$-induced change of work function is originated from interface charge redistribution, resulting in additional interface dipole moment. Our work shows a scheme to modulate the interface structure by changing the annealing gas $\mathrm{N}$ composition so as to adjust the interface effective work function, which should provide experimentalists with some insights in their effort in atomic-level chemical design of Metal/Oxide stack.

\section{Acknowledgements}

We gratefully acknowledge support of this work by the Natural Science Foundation of China (NSFC) (11202178, 11275150, 11435010) and Hunan Provincial Natural Science Foundation (14JJ3082). Works at LANL was supported by the U.S. Department

of Energy, Office of Science, Basic Energy Sciences, Materials Sciences and Engineering Division.

\section{References}

[1] J. H. Choi, Y. Mao, J. P. Chang, Development of hafnium based high-k materials-A review. Materials Science and Engineering: R, 72 (2011) 97-136. 
[2] J. G. Um, M. Mativenga, P. Migliorato, Jin Jang, Field-induced carrier generation in amorphous-InGaZnO4 thin-film transistors, Solid State Communications, 194 (2014) 54-58.

[3] S. Guha, V. Narayanan, High-k/metal gate science and technology, Annual Review of Materials Research, 39 (2009) 181-202.

[4] Y. C. Yeo, T. J. King, H. C. M. Hu, Metal-dielectric band alignment and its implications for metal gate complementary metal-oxide-semiconductor technology, Journal of Applied Physics, 92 (2002) 7266-7271.

[5] K. H. Zhong, G. G. Xu, J. M. Zhang, Z. G. Huang, Effects of strain on effective work function for Ni/HfO2 interface, Journal of Applied Physics, 116 (2014) 063707.

[6] H. Zhu, R. Ramprasad, Effective work function of metals interfaced with dielectrics: A first-principle study of the Pt-HfO2 interface, Physical Review B, 83 (2011) 081416.

[7] S. Toyoda, H. Kumigashira, M. Oshima, H. Sugaya, H. Morita, Determining factor of effective work function in metal/bi-layer high-k gate stack structure studied by phoemission spectroscopy, Applied Physics Letters, 100 (2012) 112906.

[8] X. R. Wang, Y. L. Jiang, Q. Xie, C. Detavernier, G. P. Ru, X. P. Qu, B. Z. Li, Annealing effect on the metal gate effective work function modulation for the $\mathrm{Al} / \mathrm{TiN} / \mathrm{SiO} / \mathrm{p}-\mathrm{Si}$ structure, Microelectronic Engineering, 88 (2011) 573-577.

[9] K. Xiong, J. Robertson, G. Pourtois, J. Pétry, M. Müller, Impact of incorporated Al on the TiN/HfO2 interface effective work function, Journal of Applied Physics, 104 (2008) 074501.

[10]L. P. B. Lima, H. F. Dekkers, J. G. Lisoni, J. A. Diniz, S. Van Elshocht, S. De Gendt, Metal gate work function tuning by $\mathrm{Al}$ incorporation in TiN, Journal of Applied Physics, 115 (2014) 074504.

[11]D. Gu, S. K. Dey, P. Majhi, Effective work function of Pt, Pd, and Re on atomic deposited HfO2, Applied Physics Letters, 89 (2006) 082907.

[12]A. V. Gavrikov, A. A. Knizhnik, A. A. Bagaturyants, B. V. Potapkin, M. W. Fonseca, J. Schaeffer, Oxidation of the Pt/HfO2 interface: The role of the oxygen chemical potential, Journal of Applied Physics, 101 (2007) 014310.

[13]W. Kohn, L. J. Sham, Self-consistent equations including exchange and correlation effects, Physical Review, 140 (1965) A1133.

[14]M. Fuchs, M. Scheffler, Ab initio pseudopotentials for the electronic structure calculations of poly-atomic systems using density-functional theory, Computer Physics Communications, 119 (1999) 67-98.

[15]J. P Perdew, K. Burke, M. Ernzerhof, Generalized gradient approximation made simple, Physical review letters, 77 (1996) 3865-3883.

[16]R. Ruh, P. Corfield, Crystal structure of monoclinic hafnia and comparison with monoclinic zirconia, Journal of the American Ceramic Society, 53 (1970) 126-129.

[17]http://www.webelements.com. 
[18]W.X. Li, C. Stampfl, M. Scheffler, Oxygen adsorption on $\operatorname{Ag}(111)$ : Adensity-functional theory investigation, Physical Review B, 65 (2002) 075407.

[19]X. Zhou, J. Shi, C. Li, Effects of metal doping on electronic structure and visible light absorption of $\mathrm{SrTiO}_{3}$ and $\mathrm{NaTaO} 3$ (metal $=\mathrm{Mn}, \mathrm{Fe}$, and $\mathrm{Co}$ ), The Journal of Physical Chemistry C, 115 (2011) 8305-8311.

[20]X. F. Wang, L. He, S. Halas, T. Pieńkos, J. G. Lin, T. Durakiewicz, The canonical work function-strain relationship of the platinum metal: A first-principles approach to metal-gate transistor optimization, Applied Physics Letters, 102 (2013) 223504.

[21]S. Yanagisawa, K. Lee, Y. Morikawa, First-principles theoretical study of Alq3/Al interfaces: Origin of the interface dipole, The Journal of Chemical Physics, 128 (2008) 244704.

[22]H. Wang, Y. Wang, J. Feng, C. Ye, B. Y. Wang, H. B. Wang, Q. Li, Y Jiang, A. P. Huang, Z. S. Xiao, Improved thermal stability, interface, and electrical properties of $\mathrm{HfO} 2$ films prepared by pulsed laser deposition using in situ ionized nitrogen, Applied Physics Letters, 95(2009) 032905.

[23]J. T. Sun, Y. H. Lu, W. Chen, Linear tuning of charge carriers in graphene by organic molecules and charge-transfer complexes, Physical Review B, 81 (2010) 155403.

[24]L. L. Wang, H. P. Cheng, Density functional study of the adsorption of C60 monolayer on $\operatorname{Ag}(111)$ surfaces, Physical Review B 69 (2004) 165417. 